\title{
Interference color modulation, tunable refractive index, and chiroptical electrochromism in a $\pi$-conjugated polymer with cholesteric liquid crystal order
}

\author{
Hiromasa Goto ${ }^{\text {a) }}$ \\ Graduate School of Pure and Applied Sciences, Institute of Materials Science, University of Tsukuba, \\ Tsukuba, Ibaraki 305-8573, Japan
}

(Received 5 February 2009; accepted 20 March 2009; published online 4 June 2009)

\begin{abstract}
A $\pi$-conjugated polymer film prepared by electrolytic polymerization using a cholesteric liquid crystal (CLC) is demonstrated to have a tunable interference function under electrochemical doping and dedoping. The polymer exhibits a CLC-like periodic structure with a potential-dependent refractive index and optical absorption properties. The interference color of the polymer film can thus be modulated dynamically by appropriate application of a voltage in the $\pm 1 \mathrm{~V}$ range. This research involves structural chirality and tunable chiroptical properties, doping-dedoping driven tunable refractive index, electrochromism, and interference color modulation for the present polymer. The phenomena demonstrate the possibility of electrochemical photonics. (C) 2009 American Institute of Physics. [DOI: 10.1063/1.3126524]
\end{abstract}

\section{INTRODUCTION}

Cholesteric liquid crystal (CLC) is a twisted nematic phase of liquid crystal in which the crystal directors are rotated progressively to form a helical structure. ${ }^{1}$ The helical orientation of rodlike molecules results in periodicity, and the three-dimensional molecular arrangement produces phase chirality. ${ }^{2}$ When CLC molecules are homeotropically anchored to a substrate, a periodic stripe pattern is formed corresponding to the helical half-pitch of the CLC, resulting in a visible spiral domain or fingerprint pattern. ${ }^{3}$ This periodic structure can be regarded as a one-dimensional photonic crystal $^{4,5}$ that can function as a diffraction grating by selectively reflecting light according to the half-pitch periodicity of the helical structure. This light-selective property of CLC can be modified by appropriate adjustment of the half-pitch length through external stimuli, such as electrical potential. The helicity-related optical properties of CLCs have been applied in a range of functional optical devices, including lasing devices, ${ }^{6-8}$ optical diodes, ${ }^{9}$ and optical reflectors. ${ }^{10}$

CLC has also been employed as a reaction field for the synthesis of optically active polymer films from optically inactive monomers. ${ }^{11}$ Such a CLC reaction field has successfully been employed for the preparation of polymerstabilized CLC materials. ${ }^{12,13}$ Polymer growth by this electrochemical method follows the three-dimensional chiral continuum of the CLC electrolyte solution to form a chiral architecture on the molecular level, effectively imprinting the chiral structure of the CLC field on the product polymer. ${ }^{14}$ The optically active polymer thus prepared exhibits intense optical activity, controllable circular dichroism, and optical rotation upon doping. Here, the doping level can be tuned electrochemically by appropriate application of a voltage in an electrolyte solution containing a dopant (supporting salt,

\footnotetext{
${ }^{a)}$ Electronic mail: gotoh@ims.tsukuba.ac.jp. Tel.: +81-298-53-5128. FAX: $+81-298-53-4490$.
}

perchlorate ion). Counterions are injected into the conductive polymer on the anode side, resulting in $p$-type doping. However, while the $\pi$-conjugated polymer was successfully imprinted with the molecular architecture of the CLC, the surface of the polymer film thus prepared is planar and does not exhibit iridescence.

In the present study, an optically active $\pi$-conjugated polymer film with a microperiodic convexo-concave surface $^{15}$ is prepared by a modified electrolytic polymerization method in CLC electrolyte solution from an achiral monomer with high polymerization activity. The resultant polymer film acts as a diffraction grating, displaying optical absorption and refractive index modulation that can be reversibly controlled by electrochemical doping-dedoping through the application of a low voltage. ${ }^{16}$ This polymer therefore has the functionality of interference color tuning.

\section{EXPERIMENTAL}

Polymerization was performed by an electrochemical method using a CLC electrolyte solution containing a cholesteric derivative (cholesteryl pelargonate) as a CLC inducer and the 3,4-ethylenedioxythiophene (EDOT)-based starting monomer, 1,4-bis[2-(3,4-ethylenedioxy)thienyl]phenylene (BEDOT-P). ${ }^{17}$ Here, the addition of a small amount of a chiral compound having great helical twisting power $(\beta)$ to nematic liquid crystal produces CLC with a helical superstructure. Although "chiral dopant" is the generally accepted term, "chiral inducer" is employed in this report to avoid confusion with the term "dopant" (electron accepter or donor) for conductive polymers.

The monomer was prepared in advance by Stille coupling reaction (Grignard metathesis was employed in a previous study). ${ }^{18}$ The resulting polymer (poly-BEDOT-P) was prepared on indium tin oxide (ITO) glass substrates using a sandwich cell configuration. ${ }^{11}$ 


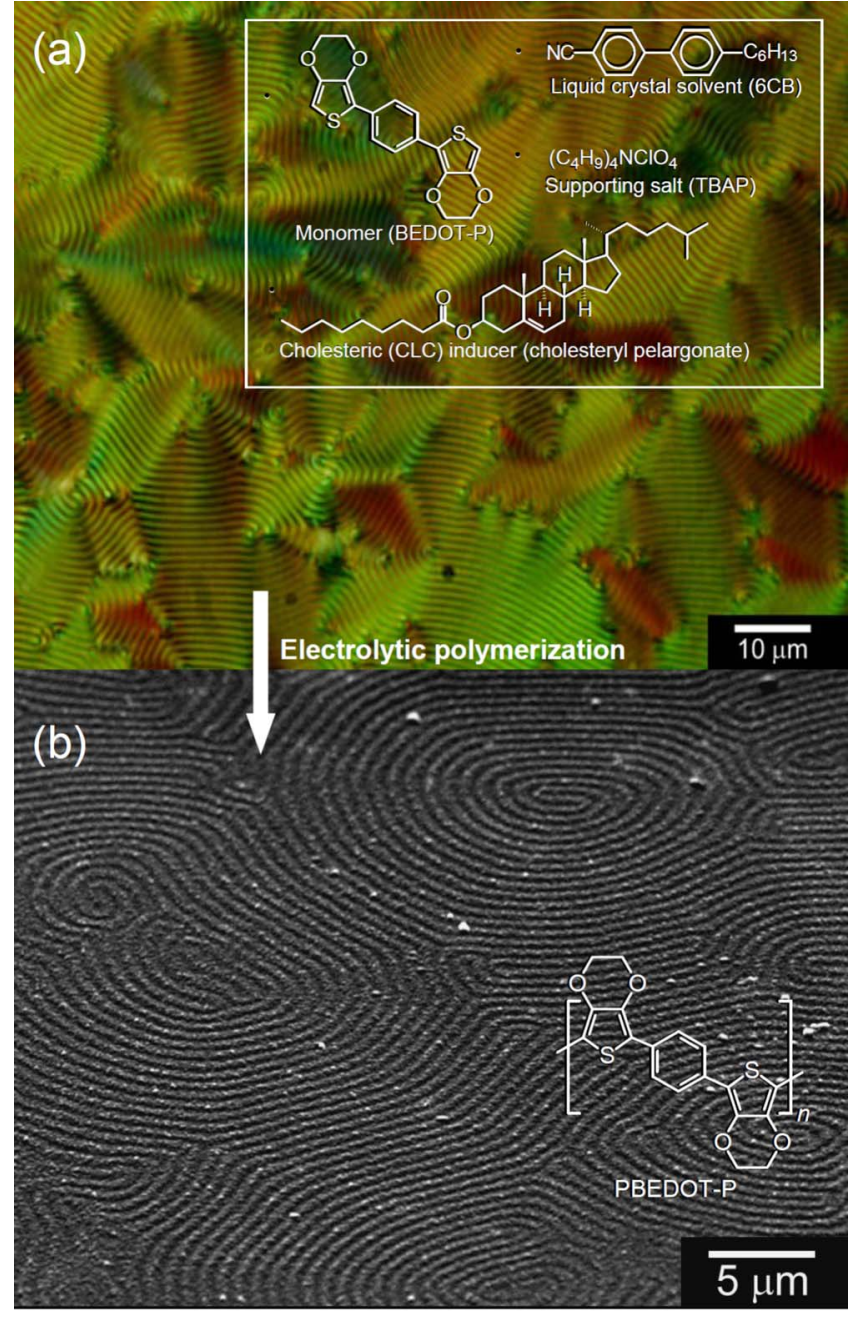

FIG. 1. (Color online) Polarizing optical microscopic image of the CLC electrolyte solution (a) and SEM images of poly-BEDOT-P (polymer) prepared by electrolytic polymerization in the CLC taken from a direction oriented $25^{\circ}$ from the surface (b).

\section{RESULTS AND DISCUSSION}

A polarizing optical microscopic (POM) image of the CLC electrolyte solution is shown in Fig. 1(a). The electrolyte solution displays the typical fingerprintlike polygonal texture of CLC materials. Scanning electron microscopy (SEM) observations reveal that the polymer prepared in the CLC exhibits a microperiodic convexo-concave surface with a fingerprintlike pattern [Fig. 1(b)]. The infrared (IR) absorption spectrum of the polymer confirms the absence of the CLC inducer (cholesteryl pelargonate, $\nu_{\mathrm{C}=\mathrm{O}}, 1739 \mathrm{~cm}^{-1}$ ) in the final polymer.

The good polymerization activity and excellent crystallinity of the polymer prepared in this study allows a singlepitch periodic pattern to be transcribed from the CLC structure. The fingerprint pattern is similar to that of typical CLC materials, although the polymer has no fluidity. The distance between stripes in the texture corresponds to the helical halfpitch $(P / 2)$ of the CLC electrolyte solution and originates from the macromolecular arrangement of the main chains in a helix matching that of the electrolyte solution. The molecular structure of the polymer, however, differs completely from that of the cholesteric electrolyte solution. The molecu-

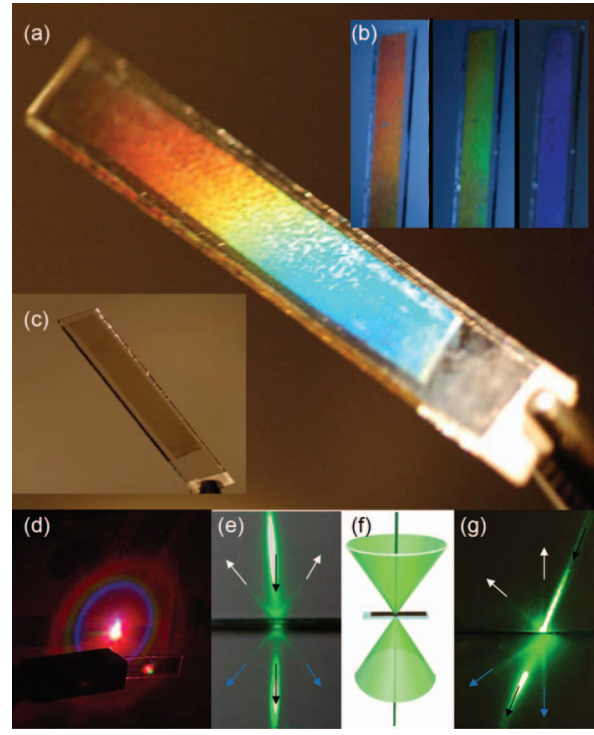

FIG. 2. (Color) [(a) and (b)] Polymer film on ITO under white light at various reflection angles. (c) Natural appearance of polymer film. (d) Diffraction pattern under irradiation with combined red, green, and blue laser light. (e) Green laser diffraction at normal incidence. Black arrow indicates incident direction, white arrows denote reflection directions, and blue arrows denote transmission directions. (f) Schematic of three-dimensional hollow cone drawn by the transmission and reflection of light at normal incidence. (g) Green laser reflection and transmission at $\theta=20^{\circ}$.

lar imprinting of the product polymer thus appears to occur by topological control of the one-handed helically twisted matrix of the CLC electrolyte solution in the course of the epitaxial electrolytic polymerization process.

The convexo-concave structure of the polymer produces two optical effects: the iridescent reflection of light and the diffraction of light. The polymer film has a rainbowlike appearance reminiscent of a jewel beetle upon irradiation at certain angles and appears monochromatic at other incident angles upon irradiation with white light from a light emitting diode [Figs. 2(a) and 2(b)]. The natural color of the polymer is dark brown in the half-doped state [Fig. 2(c)]. This reflection of light originates from the periodic convexo-concave structure of the polymer surface.

Laser transmission through the polymer film produces a circular Fourier-transformed interference figure with distinct separation of red, green, and blue laser light [Fig. 2(d)] due to the random grating formed by the single-pitch helicityderived stripes of the polymer. The red $(670 \mathrm{~nm})$, green $(532$ $\mathrm{nm})$, and blue $(473 \mathrm{~nm})$ wavelengths are projected to the outer, middle, and inner parts of the circular diffraction pattern, respectively. Upon irradiation with light at $532 \mathrm{~nm}$ from a laser diode, the polymer displays $m=1$ diffraction, as shown in Figs. 2(e) and 2(g), confirming that the polymer has both transmission and reflection grating functions. The diffraction can be expressed by the grating equation $m \lambda$ $=2 d \sin \theta$, where $d=P / 2$. Under irradiation normal to the polymer surface, the light trace draws a conic transmission and reflection pattern [Fig. 2(f)]. The three-dimensional surface of the symmetry cone can be described by a quadric cone, $x^{2} / a^{2}+y^{2} / b^{2}-z^{2} / c^{2}=0$, where $a, b$, and $c$ are constants, and $a=b \neq 0$.

Figures 3(a) and 3(b) show the transmission colors in the 

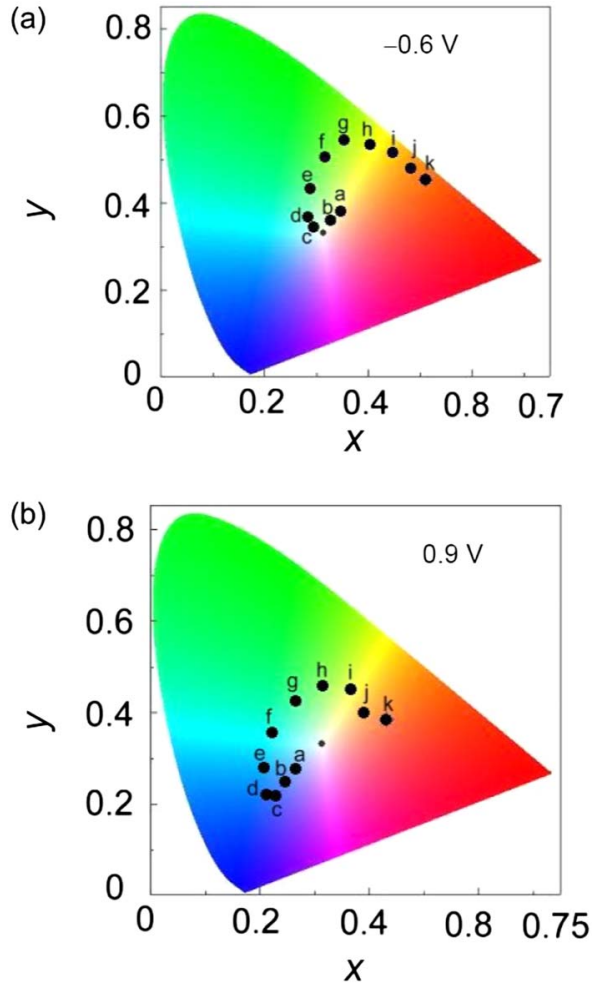

FIG. 3. (Color online) Transmission colors in CIE chromaticity space calculated from transmission spectra for normally incident light at -0.6 and $0.9 \mathrm{~V}$ vs Fc $/ \mathrm{Fc}^{+}$. Detection angles $(\theta)$ : (a) $24^{\circ}$, (b) $26^{\circ}$, (c) $28^{\circ}$, (d) $30^{\circ}$, (e) $32^{\circ}$, (f) $34^{\circ}$, (g) $36^{\circ}$, (h) $38^{\circ}$, (i) $40^{\circ}$, (j) $42^{\circ}$, and (k) $44^{\circ}$.

International Commission on Illumination (CIE) chromaticity diagram as calculated from the transmission spectra of the polymer for normally incident light at -0.6 and $0.9 \mathrm{~V}$ (versus $\left.\mathrm{Fc} / \mathrm{Fc}^{+}\right)$. The polymer exhibits blue to red colors as the detection angle $(\theta)$ is scanned from $24^{\circ}$ to $44^{\circ}$, drawing a semicircular trace. The angle-dependent profile of the color change demonstrates the selectivity of light transmission based on the diffraction property of the polymer. The color of the polymer at each angle shifts toward blue with progressive electrochemical doping.

Based on the above observations, the polymer has a CLC-like one-dimensional periodic refractive index variation, with an average refractive index $\left(n_{\mathrm{av}}\right)$ of $\left(n_{1}-n_{2}\right) / 2$ and a periodicity $(a)$ of $2 a=m \lambda / n_{\mathrm{av}}$, where $a=P / 2$. The SEM observations indicate that $P / 2$ for this polymer does not change in the course of electrochemical doping-dedoping (i.e., $P$ is constant) despite the change in the transmission wavelength.

Ellipsometry measurements reveal that the average refractive index of the polymer near $600 \mathrm{~nm}$ decreases with increasing positive voltage in the electrochemical doping process [Fig. 4(a)]. Here, the $n$ values obtained from ellipsometry measurements can be substituted for $n_{\mathrm{av}}$. The decrease in $n$ by doping results in a shortening (blueshift) of the diffraction wavelength; that is, the refractive index drives the change in the diffraction wavelength. The variation in the extinction coefficient $(\kappa)$ exhibits a similar tendency to that observed for optical absorption, namely, an increase in the applied voltage reduces absorption and the $\kappa$ intensity at 450 $\mathrm{nm}$ and strengthens the intensities at long wavelengths, as

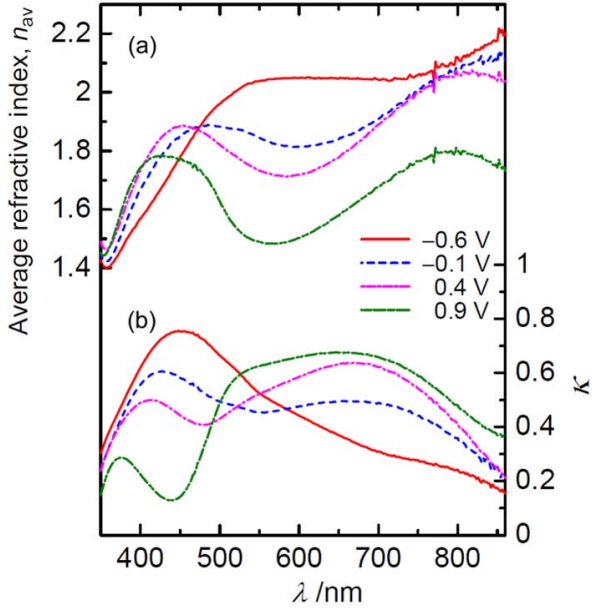

FIG. 4. (Color online) (a) Refractive index and (b) extinction coefficient for poly-BEDOT-P at $-0.6,-0.1,+0.4$, and $+0.9 \mathrm{~V}$ vs $\mathrm{Fc} / \mathrm{Fc}^{+}$at incident and detection angle of $45^{\circ}$.

shown in Fig. 4(b) ${ }^{28}$ This can be attributed to the same physical property (i.e., $\alpha=4 \pi \kappa / \lambda$; where $\alpha$ is the absorption coefficient and $\kappa \neq \alpha$ ).

Electro-optic mechanisms, such as the Kerr and Pockels effects, by which the refractive index changes with application of a voltage, are known to occur in many materials. The Frantz-Keldysh effect similarly affords a change in the optical absorption and refraction for semiconductors and insulators under an electric field. ${ }^{19-21}$ The modulation of optical properties by these processes, however, typically requires applying very high voltages. In contrast, the optical absorption and refractive index of the polymer prepared in the present study can be controlled by electrochemical doping-dedoping using an applied voltage of less than $1 \mathrm{~V}$. This can be referred to as "doping-dedoping driven change in refractive index."

The change in visible-near infrared (vis-NIR) optical absorption upon electrochemical doping-dedoping reveals an absorption maximum at $463 \mathrm{~nm}(2.7 \mathrm{eV})$, corresponding to

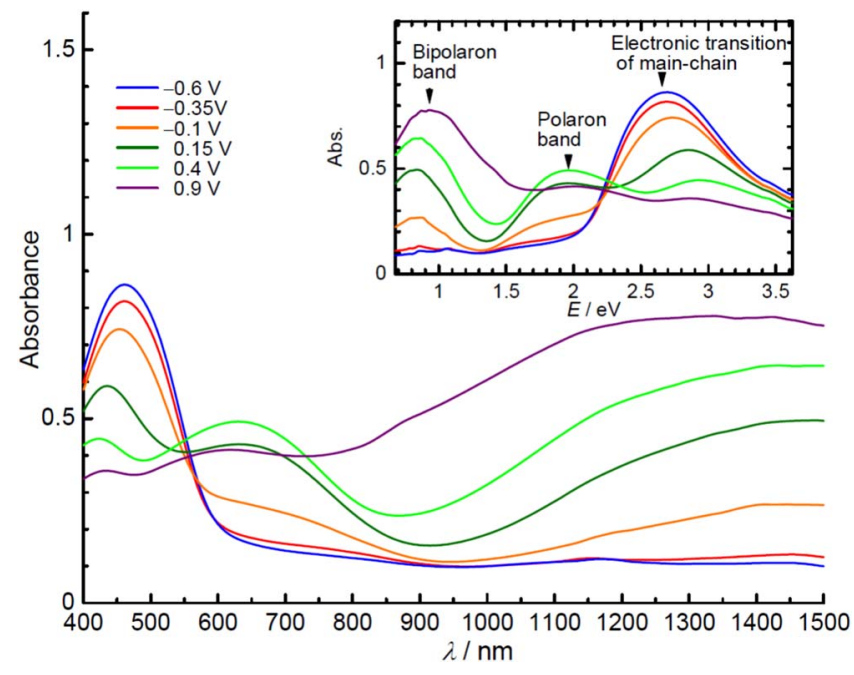

FIG. 5. (Color online) Optical absorption spectra for the polymer in $0.1 M$ TBAP/acetonitrile solution at various potentials. The inset shows absorption as a function of $E$ (wavelength is transformed into energy for the spectra). 
the $\pi$ - $\pi^{*}$ transition of the main chain (Fig. 5). Figure 5 (inset) displays absorption of the polymer as a function of energy ( $\lambda$ is transformed into energy in Fig. 5). The intensity of this peak decreases with increasing voltage, accompanied by the emergence of a new absorption maximum at $639 \mathrm{~nm}(1.9$ $\mathrm{eV}$ ) and an absorption band in the red to NIR region ( $<1 \mathrm{eV}$ ) attributable to the generation of polarons and bipolarons on the $\pi$-conjugated main chains, respectively. ${ }^{22-25}$ The natural color appearance in the CIE chromaticity diagram as calculated from the optical absorption spectra of the polymer at -0.6 and $0.9 \mathrm{~V}$ indicates that the color of the polymer at each applied voltage shifts toward blue with progressive electrochemical doping. This result corresponds to that of the transmission CIE result.

The transmission intensity at a certain wavelength $\left(\lambda_{1}\right)$ can be expressed by $I_{\text {tot }}=I_{0}-I_{\text {abs }}-I_{\text {ref }}$, where $I_{\text {tot }}$ is the total transmission intensity, $I_{0}$ is the input intensity, $I_{\text {abs }}$ is the absorption intensity, and $I_{\text {ref }}$ is the reflection intensity. The $I_{0}-I_{\text {abs }}$ component can be obtained using $I_{0} e^{-\alpha x}$, where $\alpha$ is the absorption coefficient according to the Lambert-Beer law. Hence, $I$ is primarily dependent on the optical absorption of the polymer. The generation of polarons as an electrical conduction carrier accompanying doping strengthens optical absorption in the red wavelength region, resulting in overall enhancement of transmission in the blue region.

The modulation of light using materials with tunable optical absorption properties can be explained in terms of the change in the complex index of refraction $(N)$, which is given by $N=n-i \kappa$. Doping causes a reduction in the refractive index $n$ of the polymer by the electro-optic effect and an increase in $-i \kappa$ at long wavelengths (corresponding to gain loss) by absorption change. Doping therefore drives the total refractive index.

In the doping process for the polymer, the value of $n$ in the green-orange region is reduced and the optical absorption intensity in the red region is increased, which enhances the transmission of the blue wavelength region light as a complementary color. Furthermore, the reduction in the absorption intensity in the blue region allows transmission of blue light from the polymer. In other words, the generation of the polarons on the polymer by the doping changes the electronic structure of the polymer. This change affords change in refractive index and absorption at a certain detection angle. The change in the color is reversible with the sequential electrochemical doping-dedoping process. This can be referred to as an interference color modulation electrochromism based on tunable transmission effect for the electroactive polymer with diffraction property.

The change in surface color of the present polymer during redox cycling is displayed in Fig. $6(-0.6$ and +0.9 V). Under obliquely incident white light, the natural color of the polymer changes from dark orange to mazarine according to the oxidation state, while the structural color changes from light blue to dark interference colors.

Optical rotatory dispersion (ORD) for the polymer at three applied voltage in $0.1 M$ tetrabutyl ammonium perchlorate (TBAP)/acetonitrile solution is shown in Fig. 7. Upon doping (increase in the applied voltage), an optical rotation at short wavelengths and a broad optical rotation signal at
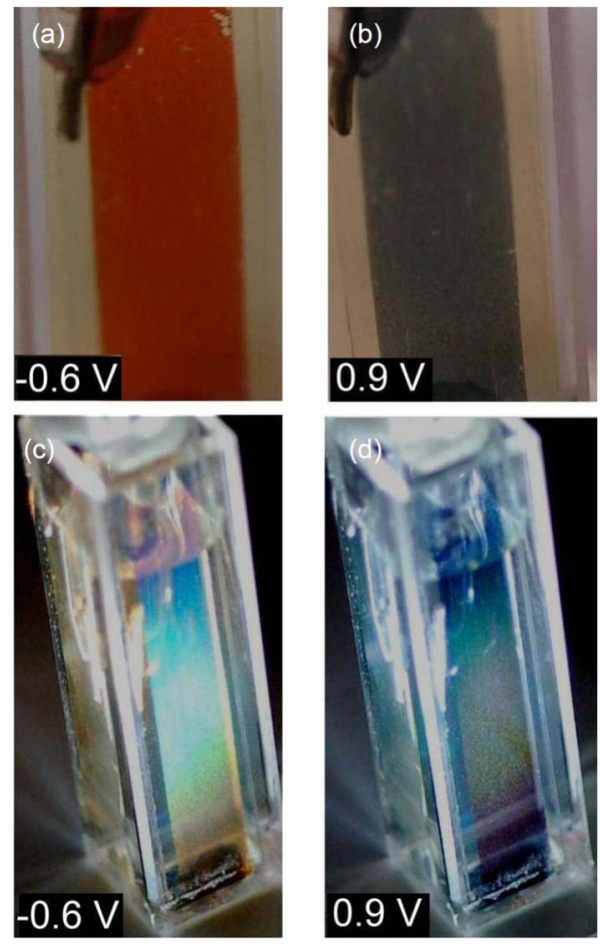

FIG. 6. (Color online) Electrochromism and reflection interference electrochromism of the polymer in $0.1 M$ TBAP/acetonitrile solution at -0.6 and $+0.9 \mathrm{~V}$ vs $\mathrm{Fc} / \mathrm{Fc}^{+}$. [(a) and (b)] Natural appearance and [(c) and (d)] structural color under obliquely incident white light.

long wavelengths with an isosbestic point of $490 \mathrm{~nm}$. This result indicates that the polymer is electrochiroptically active and that the optical rotation can be tuned via the electrochemical doping process. This property of the polymer can be regarded as an "electrochemically driven chiroptical effect." The polymer produces a maximum optical rotation of $-2.2 \times 10^{4} \mathrm{~cm} / \mathrm{deg}$ at $445 \mathrm{~nm}$. These values are comparable to the Faraday rotation of magnetic transition-metal materials such as $\mathrm{Fe}\left(+3.8 \times 10^{5} \mathrm{deg} / \mathrm{cm}\right),{ }^{25}$ and $\mathrm{NdFeO}_{3}(+4.7$ $\left.\times 10^{4} \mathrm{deg} / \mathrm{cm}\right)^{26,27}$ under POM observations. This result indicates that structural chirality produced by cholesterics effectively provides chiral architecture for the polymer. The optical rotations of the polymer during voltage scanning from -0.1 to $+0.9 \mathrm{~V}$ undergo reversible changes at $440 \mathrm{~nm}$ (Fig. 7, inset). This change in optical rotation demonstrates reversible chiroptical electrochromism.

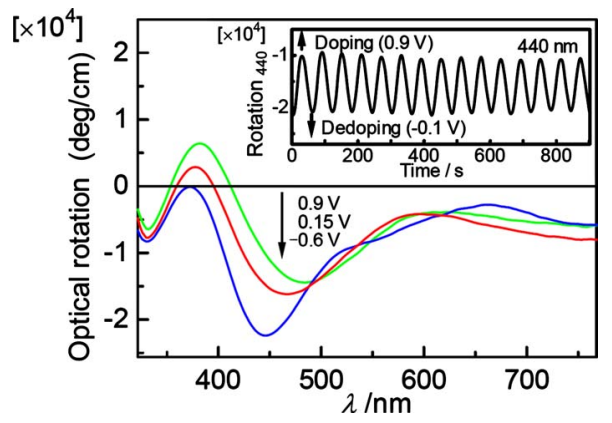

FIG. 7. (Color online) ORD spectra for the polymer on ITO electrode in TBAP/acetonitrile solution at three voltages vs $\mathrm{Fc} / \mathrm{Fc}^{+}$. The inset shows reversible change in the optical rotation at $440 \mathrm{~nm}$ with electrochemical doping-dedoping cycle. 


\section{CONCLUSION}

The electroactive polymer, poly(1,4-bis[2-(3,4ethylenedioxy)thienyl]phenylene), prepared by an electrochemical method using a CLC electrolyte solution was demonstrated to have a molecular helical structure transcribed from the CLC. As a result of its helical structure, the polymer film exhibits characteristic diffraction that can be tuned by electrochemical doping and dedoping. The diffraction function originates from the CLC-like periodic structure, and the diffraction color is determined by the refractive index and optical absorption, both of which are changed by doping/ dedoping due to the generation/loss of polarons on the polymer main chain and the resultant change in the electronic structure. The optical properties of the polymer are thus dynamically tunable by appropriate application of a voltage in the $\pm 1 \mathrm{~V}$ range. This electrochromic effect, a type of interference color modulation determined by potential-dependent diffraction, is demonstrative of the possibility of a new field of "electrochemical photonics."

\section{ACKNOWLEDGMENTS}

The author would like to thank the chemical analysis center and the glass workshop of the University of Tsukuba.

${ }^{1}$ I. Dierking, Textures of Liquid Crystals (Wiley VCH, New York, 2003).

${ }^{2}$ S. Courty, A. R. Tajbakhsh, and E. M. Terentjev, Eur. Phys. J. E 12, 617 (2003).

${ }^{3}$ P. Oswald and A. Dequidt, Phys. Rev. E 77, 051706 (2008).

${ }^{4}$ I. I. Smalyukh, B. I. Senyuk, P. Palffy-Muhoray, O. D. Lavrentovich, H. Huang, E. C. Gartland, Jr., V. H. Bondnar, T. Kosa, and T. Taheri, Phys. Rev. E 72, 061707 (2005).

${ }^{5}$ N.-Y. Ha, S.-M. Jeong, S. Nishimura, G. Suzaki, K. Ishikawa, and H.
Takezoe, Adv. Mater. (Weinheim, Ger.) 20, 2503 (2008).

${ }^{6}$ K. Yoshino, H. Takeda, M. Kasano, S. Satoh, T. Matsui, R. Ozaki, A. Fujii, M. Ozaki, and A. Kose, Macromol. Symp. 212, 179 (2004).

${ }^{7}$ R. Ozaki, T. Matsui, M. Ozaki, and K. Yoshino, Electron Commun. Jpn., Part 2: Electron. 87, 1 (2004)

${ }^{8}$ Y. Zhou, Y. Huang, Z. Ge, L.-P. Chen, Q. Hong, T.-X. Wu, and S.-T. Wu, Phys. Rev. E 74, 061705 (2006).

${ }^{9}$ J. Hwang, M.-H. Song, B. Park, S. Nishimura, T. Toyoda, J. W. Wu, Y. Takanishi, K. Ishikawa, and H. Takezoe, Nature Mater. 4, 383 (2005).

${ }^{10}$ M.-Y. Ha, Y. Ohtsuka, S.-M. Jeong, S. Nishimura, G. Suzaki, Y. Takanishi, K. Ishikawa, and H. Takezoe, Nature Mater. 7, 43 (2008).

${ }^{11}$ H. Goto, Phys. Rev. Lett. 98, 253901 (2007).

${ }^{12}$ I. Dierking, L. L. Kosbar, A. Afzali-Ardakani, A. C. Lowe, and G. A. Held, J. Appl. Phys. 81, 3007 (1997).

${ }^{13}$ G. A. Held, L. L. Kosbar, I. Dierking, A. C. Lowe, G. Grinstein, V. Lee, and R. D. Miller, Phys. Rev. Lett. 79, 3443 (1997).

${ }^{14}$ H. Goto, J. Electrochem. Soc. 154, E63 (2007).

${ }^{15} \mathrm{H}$. Yoneyama, K. Kawabata, A. Tsujimoto, and H. Goto, Electrochem. Commun. 10, 965 (2008).

${ }^{16}$ H. Goto, Japan Patent No. 200732790 (13 February 2007); Chem. Abstr. 149, 379216 (2008); H. Goto, Adv. Funct. Mater. 19, 1 (2009).

${ }^{17}$ A. Tsujimoto and H. Goto, Mol. Cryst. Liq. Cryst. 493, 82 (2008).

${ }^{18}$ G. Sotzing and J. R. Reynolds, Chem. Mater. 8, 882 (1996).

${ }^{19}$ W. Frantz, Z. Naturforsch. A 13A, 484 (1958).

${ }^{20}$ L. V. Keldysh, Sov. Phys. JETP 20, 1307 (1965).

${ }^{21}$ P. Williams, Phys. Rev. 117, 1487 (1960).

${ }^{22}$ M. Walczak and J. R. Reynolds, Adv. Mater. (Weinheim, Ger.) 18, 1121 (2006).

${ }^{23}$ J. Heeger, S. Kivelson, J. R. Schrieffer, and W.-P. Su, Rev. Mod. Phys. 60, 781 (1988)

${ }^{24}$ Y. Furukawa, J. Phys. Chem. 100, 15644 (1996).

${ }^{25}$ F. Diaz, J. I. Castillo, J. A. Logan, and W.-Y. Lee, J. Electroanal. Chem. Interfacial Electrochem. 129, 115 (1981).

${ }^{26}$ W. A. Crossley, R. W. Cooper, J. L. Page, and R. P. van Staple, Phys. Rev. 181, 896 (1969).

${ }^{27}$ K. Sato, Light and Magnetism (Asakura, Tokyo, Japan, 2003), Vol. 13

${ }^{28}$ See EPAPS Document No. E-JAPIAU-105-153909 for change in $\delta$ and $\psi$ value at the applied voltages obtained with ellipsometry measurement. For more information on EPAPS, see http://www.aip.org/pubservs/epaps.html. 